\title{
Glycated hemoglobin versus oral glucose tolerance test in the identification of subjects with prediabetes in Qatari population
}

Saadallah Iskandar ${ }^{1}$, Ayman Migahid², Dalia Kamal², Osama Megahed ${ }^{2}$, Ralph A. DeFronzo ${ }^{3}$, Mahmoud Zirie $^{2}$, Amin Jayyousi ${ }^{2}$, Mahmood Al Jaidah ${ }^{1}$ and Muhammad Abdul-Ghani ${ }^{2,3^{*}}$ (D)

\begin{abstract}
Background: Subjects with prediabetes are at increased risk of future T2DM and cardiovascular disease (CVD) compared to NGT individuals. The OGT (FPG $=100-125$ and $2 \mathrm{~h}-\mathrm{PG}=140-199 \mathrm{mg} / \mathrm{dl}$ ) and HbA1c 5.7-6.4\% have been used to diagnose subjects with prediabetes. In the present study, we compared the ability of the OGTT and HbA1c to identify Qatari subjects with prediabetes.

Methods: Four hundred forty six subjects without a history of T2DM received 75-g OGTT and measurement of $\mathrm{HbA1c}$. The incidence of prediabetes in this cohort according to OGTT criteria was compared to that of HbA1c criteria.

Results: The agreement between the OGTT and HbA1c in identifying subjects with prediabetes in Qatari subjects was poor, though significant $(k=015, p<0.0001)$. Only $56 \%$ of participants had prediabetes or NGT according to OGTT and $\mathrm{HbA1c}$. The disagreement between OGTT and HbA1c in diagnosing prediabetes was primarily due to low sensitivity of $\mathrm{HbA1c}$. Moreover, subjects with prediabetes diagnosed with the OGT have more severe metabolic profile than prediabetic subjects diagnosed with HbA1c. Lastly, more subjects with the metabolic syndrome were identified with OGTT (60\%) criteria than with the HbA1c (49\%), $p<0.0001$.
\end{abstract}

Conclusion: These results demonstrate subjects with prediabetes diagnosed with OGTT have more severe metabolic risk than those diagnosed with $\mathrm{HbA1c}$, and more likely to have greater risk of progression to T2DM.

Keywords: Prediabetes, Type 2 diabetes, HbA1c, OGTT

\section{Background}

The prevalence of type 2 diabetes mellitus (T2DM) is increasing at epidemic rates worldwide and the diabetes epidemic is even more widespread in the Middle East [1-3]. The prevalence of T2DM exceeds $20 \%$ in many Gulf countries and an additional $\sim 20 \%$ of the population is at increased future risk, i.e. prediabetes $[4,5]$. In Qatar, 16.5\% manifest T2DM and approximately similar number has prediabetes [4]. Thus, approximately one

\footnotetext{
* Correspondence: abdulghani@uthscsa.edu

${ }^{2}$ Academic Health System, Hamad General Hospital, PO Box 3050, Doha, Qatar

${ }^{3}$ Diabetes Division, University of Texas Health Science Center, San Antonio, TX, USA

Full list of author information is available at the end of the article
}

third of adults Qatari individuals have prediabetes or established diabetes. Both lifestyle change and pharmacotherapy have been shown to reduce the risk of progression to T2DM in high risk individuals [6-8]. Thus, identification of high risk individuals is a key for diabetes prevention and restraining the epidemic spread of the disease [9].

Because subjects with impaired fasting glucose (IFG) and/or impaired glucose tolerance (IGT) manifest greater risk of progression to T2DM than subjects with normal glucose tolerance (NGT) [10], both IFG and IGT are considered to be prediabetes states by the American Diabetes Association [11], and all intervention studies which have assessed the efficacy of various intervention

(c) The Author(s). 2019 Open Access This article is distributed under the terms of the Creative Commons Attribution 4.0 International License (http://creativecommons.org/licenses/by/4.0/), which permits unrestricted use, distribution, and reproduction in any medium, provided you give appropriate credit to the original author(s) and the source, provide a link to the Creative Commons license, and indicate if changes were made. The Creative Commons Public Domain Dedication waiver (http://creativecommons.org/publicdomain/zero/1.0/) applies to the data made available in this article, unless otherwise stated. 
strategies have recruited subjects with IGT, IFG, or IFG/ IGT [6-8]. Because the diagnosis of IGT requires an oral glucose tolerance test (OGTT), the International Diabetes Federation has suggested that the diagnosis of prediabetes be made based upon the HbA1c since it does not require an OGTT and can be measured under nonfasting conditions [12]. HbA1c between 5.7 to $6.4 \%$ has been identified as the diagnostic range of prediabetes. Studies which have compared the ability of HbA1c versus OGTT to identify subjects with prediabetes [13-18] have reported discordance between the two methods. Further, the degree of agreement between the two methods varies amongst various ethnic groups [15, 17]. Accurate identification of high risk individuals is essential for any intervention program, since it lowers the number of subjects enrolled in the intervention program and increases the cost effectiveness of the intervention program. The state of Qatar is considering a National Diabetes Strategy for diabetes prevention based upon the identification of high risk individuals using the HbA1c. Because of the disagreement between the diagnosis of prediabetes based upon HbA1c criteria versus the OGTT and the ethnic dependence of the concordance between both methods, it is important to determine the optimal test and the appropriate cut points for detection of subjects with prediabetes in Qatari population. The aim of the present study is to compare the HbAlc versus the OGTT in the identification of Qatari subjects with prediabetes and the underlying metabolic abnormalities associated with prediabetes.

\section{Methods}

The present study was a retrospective analysis of data collected in healthy subjects, without a history of T2DM, who are seeking employment opportunity at Qatar Petroleum. Each subject seeking employment at Qatar Petroleum receives a comprehensive physical examination, lab work and 75-g OGTT to ensure that he is in a good health. Subjects who fulfilled the following criteria were included in the analysis: 1) Arabic origin; (2) no history of T2DM; (3) had HbA1c $<6.5 \%$, and FPG $<126 \mathrm{mg} / \mathrm{dl}$ and 2-h plasma glucose $<200 \mathrm{mg} / \mathrm{dl}$; (4) general good health as indicated by $\mathrm{CBC}$, blood chemistry and thyroid function.

Four hundred forty six subjects were eligible for the analysis. Medical history, physical examination, blood chemistries, lipid profile, and HbA1c were measured. The clinical and metabolic characteristics of the subjects are shown in Table 1. None of the subjects were taking any medications known to affect glucose tolerance.

The OGTT was performed in the morning after a 10$12 \mathrm{~h}$ overnight fast. Blood samples were collected before and $2 \mathrm{~h}$ after drinking $75 \mathrm{~g}$ glucose in $300 \mathrm{ml}$ of orange flavored solution. Plasma glucose concentration and
Table 1 Baseline characteristics of subjects with prediabetes and NGT

\begin{tabular}{|c|c|c|c|}
\hline & NGT & $\mathrm{IFG} / \mathrm{IGT}$ & $P$ Value \\
\hline Number & 150 & 296 & \\
\hline Age (years) & $45 \pm 1$ & $44 \pm 1$ & NS \\
\hline Gender (females) & $21(14 \%)$ & $55(19 \%)$ & NS \\
\hline BMI $\left(\mathrm{kg} / \mathrm{m}^{2}\right)$ & $30.3 \pm 0.4$ & $29.6 \pm 0.3$ & NS \\
\hline Waist (cm) & $99.6 \pm 0.7$ & $101.3 \pm 0.8$ & NS \\
\hline Positive Family History & $62 \%$ & $64 \%$ & NS \\
\hline $\mathrm{FPG}(\mathrm{mM})$ & $5.22 \pm 0.04$ & $5.94 \pm 0.04$ & $<0.0001$ \\
\hline 2-h PG (mM) & $5.78 \pm 0.08$ & $7.60 \pm 0.14$ & $<0.0001$ \\
\hline $\mathrm{HbA} 1 \mathrm{c}(\%)$ & $5.52 \pm 0.03$ & $5.67 \pm 0.02$ & $<0.0001$ \\
\hline Systolic Blood Pressure & $125 \pm 1$ & $129 \pm 1$ & 0.01 \\
\hline Total Cholesterol (mM) & $4.7 \pm 0.1$ & $4.9 \pm 0.1$ & NS \\
\hline Triglycerides (mM) & $1.34 \pm 0.07$ & $1.66 \pm 0.08$ & 0.04 \\
\hline $\mathrm{HDL}(\mathrm{mM})$ & $1.11 \pm 0.03$ & $1.09 \pm 0.06$ & NS \\
\hline
\end{tabular}

$B M I$ Body mass index, FPG fasting plasma glucose, 2-h PG 2-h plasma glucose concentration

serum insulin were measured during the fasting state and at 2-h.

The study protocol was approved by Hamad Medical Corporation IRB board (HMC Ethical committee) and informed written consent was obtained from participants prior to enrollment.

\section{Analytical methods}

Glucose was measured with the glucose oxidase method. The variability of the measurement was $2.7 \%$. HbA1c was measured by high pressure liquid chromatography (HPLC) (Knauer HPLC, Advanced Scientific Instruments, Germany). Blood pressure was measured with Digital Omni which is user independent, in the sitting position after $10 \mathrm{~min}$ of rest and represents the mean of 3 values.

\section{Calculations and statistical analysis}

Definitions: prediabetes was designed as $\mathrm{HbA1c}=5.7-$ $6.4 \%$, and subjects with $\mathrm{HbA} 1 \mathrm{c}<5.7 \%$ were considered having normal glucose tolerance. The ADA criteria of prediabetes were used to diagnosed prediabetes with OGTT (i.e. $F P G=100-125 \mathrm{mg} / \mathrm{dl}$ and/or 2-h plasma glucose $=140-199 \mathrm{mg} / \mathrm{dl}$ ).

Insulin sensitivity and insulin secretion indices were calculated with Homeostatsis Model Assessment, HOMA-IR and HOMA-B as previously described [19]. Beta cell function was measured as the product of insulin secretion (measured with HOMA-B) and (1/HOMA-IR). The ATPIII criteria for the metabolic syndrome [20] were utilized to diagnose the presence of the metabolic syndrome.

To estimate the future T2DM risk of study participants, the multivariate logistic prediction model described by 
Stern et al. [21] was utilized. This model estimates the 78 year diabetes risk and has been validated in multiple prospective population studies with $80 \%$ sensitivity and $78 \%$ specificity.

The $\mathrm{k}$-coefficient was used to test for agreement between A1C categorization of subjects and OGTT-based diagnoses of normal glucose tolerance and prediabetes.

\section{Results}

One hundred fifty out of 446 participants (34\%) had NGT and 296 subjects (66\%) had prediabetes (IFG and/ or IGT) according to the ADA criteria [22]. Table 1 presents the baseline characteristics of subjects with prediabetes and NGT. Both groups were comparable in age, gender, BMI and family history of T2DM. NGT subjects had a significantly lower HbA1c, FPG and 2-h plasma glucose concentration compared to subjects with prediabetes.

Two hundred subjects (45\%) had an HbA1c $=5.7-$ $6.4 \%$ and, therefore, were considered to have prediabetes according to this parameter $(p=0.0007$ versus OGTT criteria). The other 246 participants had HbA1c $<5.7 \%$ and, therefore, were considered normal according to HbA1c criteria $(p<0.001$ versus OGTT criteria). There was large discordance in the diagnosis of prediabetes and NGT diagnosed with OGTT versus HbA1c criteria (Table 2). Only 150 subjects were diagnosed with prediabetes according to both criteria and 100 subjects had NGT according to both criteria. Thus, 196 subjects (44\%) had the diagnosis of prediabetes or NGT according to one criteria and the opposite diagnosis according to the other criteria. Therefore, the agreement between OGTT and HbA1c in diagnosing glucose tolerance was low, though significant $(\mathrm{k}=0.15, p<0.0001)$. Further, there was a weak, though significant, correlation between the HbA1c and FPG $(\mathrm{r}=0.16, p=0.01)$ (Fig. 1) and between the HbA1c and 2-h plasma glucose concentration $(\mathrm{r}=0.19, p<0.001)$ (Fig. 2).

Previous studies $[13,18]$ have reported interaction between BMI and the concordance between HbA1c and OGTT in the diagnosis of glucose tolerance. In the present study we failed to observe any interaction between the diagnosis of glucose tolerance and either BMI or gender. The concordance between the two sets of criteria was 51, 53 and $59 \%$ in subjects with $\mathrm{BMI}<27,27-$ 30 and $>30 \mathrm{~kg} / \mathrm{m}^{2}$, respectively ( $p=\mathrm{NS}$ ), while the concordance was $50 \%$ in females and $57 \%$ in males $(p=\mathrm{NS})$.

Because HbA1c and OGTT defined distinct populations of high risk individuals, we have compared the metabolic profile of subjects classified based upon OGTT versus HbA1c. Impaired beta cell function is the principal factor responsible for the increased future T2DM risk [23]. Further, subjects at risk of T2DM manifest impaired beta cell function long before T2DM become evident. Therefore, we compared beta cell function in subjects diagnosed with and without prediabetes with HbA1c and OGTT criteria. Compared to NGT, subjects with prediabetes had significantly lower beta cell function whether prediabetes was diagnosed with $\mathrm{HbA1c}$ (by $11 \%, p<0.01$ ) or with OGTT (by $37 \%, p<0.0001$ ) (Table 3). However, beta cell function in subjects diagnosed as NGT with the HbA1c was significantly lower (by $21 \%, p<0.0001$ ) than in NGT subjects diagnosed with OGTT. Conversely, subjects diagnosed as prediabetes with HbA1c had significantly higher beta cell function (by $13 \%, p<0.0001$ ) than subjects diagnosed with prediabetes with the OGTT. Moreover, beta cell function in subjects with prediabetes diagnosed with OGTT criteria but with $\mathrm{HbA1c}<5.7 \%$ (i.e., NGT according to HbA1c criteria) was comparable to that in subjects with prediabetes according to both sets of criteria. Conversely, subjects with prediabetes according to HbA1c criteria but with NGT according to OGTT had comparable beta cell function to that of NGT subjects diagnosed with both sets of criteria (Table 4). Further, the metabolic profile of subjects with prediabetes diagnosed with the OGTT only was more severe than the profile of those diagnosed with HbA1c alone (Table 4). Consistent with this, the probability of future T2DM estimated with the Stern model in subjects diagnosed with prediabetes by the OGTT was 2.2-fold greater than in NGT subjects, compared to only $49 \%$ higher in subjects with prediabetes diagnosed with the HbA1c (last row Table 4).

Lastly, the prevalence of metabolic syndrome was 3fold greater in subjects with prediabetes than in NGT subjects according to the OGTT criteria, while it was

Table 2 Prevalence of subjects with NGT and prediabetes diagnosed with OGTT and HbA1c

HbA1c Criteria

$\underline{\text { NGT }} \quad \underline{\text { Prediabetes }}$

OGTT

Criteria
NGT

Prediabetes
100

146
50

150 


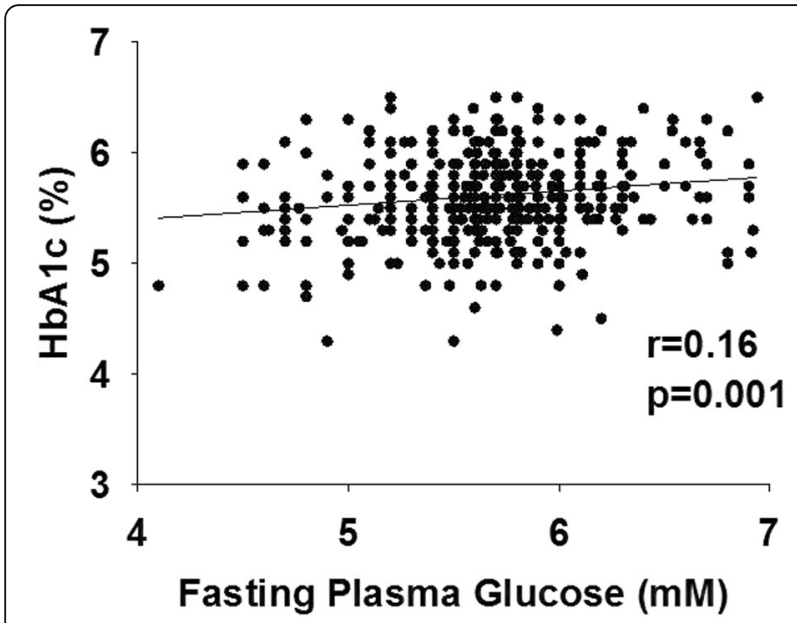

Fig. 1 Relationship between $\mathrm{HbA} 1 \mathrm{c}$ and the fasting plasma glucose concentration in subjects with NGT and prediabetes

similar in subjects with prediabetes and NGT according to HbA1c criteria.

\section{Discussion}

The results of the present study demonstrate that the concordance between the two sets of criteria (OGTT and HbA1c) utilized to identify subjects at high future T2DM risk, i.e. prediabetes, is poor in Qatari subjects. Only $56 \%$ of subjects were diagnosed with NGT or prediabetes with both sets of criteria. The other $44 \%$ were diagnosed with prediabetes with one set of criteria and NGT with the other. The disagreement between OGTT and $\mathrm{HbA} 1 \mathrm{c}$ was primarily due to lower sensitivity of HbA1c in identifying all subjects with IFG and/or IGT. These results are consistent with several previous studies in other ethnic groups [13-18] which also demonstrated low concordance between HbAlc and OGTT in

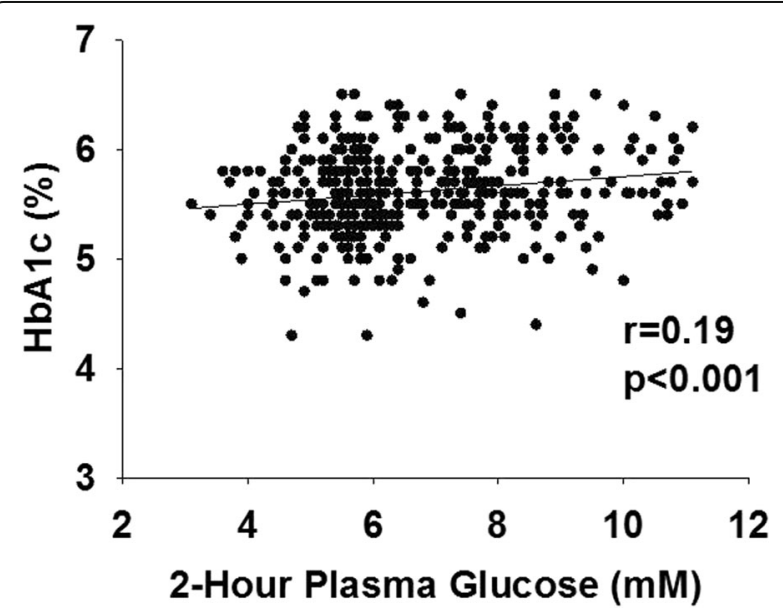

Fig. 2 Relationship between $\mathrm{HbA} 1 \mathrm{c}$ and 2-h plasma glucose concentration in subjects with NGT and prediabetes diagnosing prediabetes, and the high risk subjects identified by each set of criteria are distinct. Further, subjects with prediabetes identified with the OGTT alone had more sever metabolic profile than subjects identified with HbA1c alone, and are more likely to progress to T2DM (Table 4). Suggesting that in this population, the OGTT is a better tool in identifying subjects at high risk of T2DM, i.e. prediabetes. The results of the present study also are consistent with previous study which demonstrated low sensitivity of $\mathrm{HbA1c}$ in identifying subjects with prediabetes diagnosed with OGTT in Arab Americans [24]. Accurate identification of subjects at increased future T2DM risk is important for diabetes prevention programs, reduces the number needed to treat and improves the cost effectiveness of the intervention program. The results of the present study demonstrate that, in the Qatari population, the OGTT identifies a larger number of subjects with prediabetes than the HbA1c, i.e. higher sensitivity. Stated otherwise, many IFG and/or IGT subjects who are at increased diabetes risk and who would have benefited from the intervention program have an HbAlc $<5.7 \%$ and, thus, would not have been identified using the HbA1c criteria. These findings are consistent with previous diabetes prevention studies [6-8] in which mean baseline HbA1c in subjects diagnosed as prediabetes with the OGTT was $\sim 5.7-5.9 \%$, indicating that many subjects with an HbA1c $<5.7 \%$ have prediabetes and would benefit from an intervention program.

We and others [9, 10, 23, 25] previously have shown that OGTT criteria have more sensitivity and specificity in identifying subjects at increased future T2DM compared to HbA1c criteria. Because there was large discordance between the prevalence of prediabetes diagnosed with HbA1c versus OGTT criteria in the present study, and longitudinal data is unavailable in this population, we utilized diabetes prediction models [21] to identify subjects at increased risk of diabetes and compare the future risk of diabetes in subjects diagnosed with each set of criteria. Further, many previous intervention studies have demonstrated that subjects at high risk of diabetes manifest impaired beta cell function long before T2DM is evident $[10,23]$, and the impairment in beta cell function was the strongest predictor of future T2DM risk. Because of lack of "gold standard" for identification of who is really at increased future T2DM risk, we utilized the risk score, and impairment in beta cell function as "surrogates" to compare the actual diabetes risk in subjects diagnosed with prediabetes according to OGTT and HbA1c criteria. The results of the present study have demonstrated that the OGTT is a better tool to identify subjects with higher score of Stern model (Table 3) and lower beta cell function (Table 4) than HbA1c. In other words, a subject with $\mathrm{Hb} 1 \mathrm{Ac}<5.7 \%$ 
Table 3 Beta cell function (measured with HOMA Model Assessment [19], and T2DM risk measured with the risk score developed by Stern et al. [21] and prevalence of the metabolic syndrome (according to ATPIII criteria [20] in NGT and subjects with prediabetes diagnosed with the OGTT and $\mathrm{HbA1C}$

\begin{tabular}{|c|c|c|c|c|c|c|}
\hline & \multicolumn{3}{|c|}{ HbA1c Criteria } & \multicolumn{3}{|c|}{ OGTT Criteria } \\
\hline & Normal & Prediabetes & $P$-Value & Normal & Prediabetes & $P$-Value \\
\hline Number & 246 & 200 & & 150 & 296 & \\
\hline Beta Cell Function & $42 \pm 1$ & $37 \pm 1$ & 0.001 & $53 \pm 2$ & $33 \pm 1$ & $<0.0001$ \\
\hline$\%$ With MS & 44 & 49 & NS & 20 & 60 & $<0.0001$ \\
\hline Future T2DM Risk Score & $12.9 \pm 0.8$ & $19.3 \pm 1.2$ & $<0.0001$ & $8.8 \pm 0.5$ & $19.3 \pm 1.1$ & $<0.0001$ \\
\hline
\end{tabular}

MS metabolic syndrome

who has IFG/IGT manifest greater chance of having high score in Stern model for future T2DM and impaired beta cell function than subject who has NGT (according to OGTT) and $\mathrm{HbA} 1 \mathrm{c}=6.0 \%$.

Our results demonstrate that beta cell function in subjects diagnosed with prediabetes based upon the $\mathrm{HbA1c}$ (i.e. 5.7-.6.4\%) but with NGT based on the OGTT is comparable to that in NGT subjects (Table 4), suggesting that relying on the $\mathrm{HbA1c}$ alone to identify high risk individuals will result in the inclusion of many subjects with normal beta cell function who actually are at low future T2DM risk (i.e., false positives). These results are consistent with our previous findings in Mexican Americans [24].

Third, many previous studies $[25,26]$ have demonstrated that subjects who fulfill the criteria of the metabolic syndrome manifest greater risk of cardiovascular disease (CVD) than subjects without the metabolic syndrome. The results of the present study demonstrate that prediabetes diagnosed with the OGTT is a stronger predictor of the metabolic syndrome and, therefore, subsequent CVD risk, than the HbA1c alone. Because the primary aim of diagnosing prediabetes is to identify the subgroup of subjects who are at increased future risk for T2DM and CVD, these findings indicate that, in Qatari subjects, the OGTT should be favored over the HbA1c for the identification of subjects with prediabetes.

We previously $[27,28]$ demonstrated that the $1-\mathrm{h}$ plasma glucose concentration during the OGTT is the strongest predictor of future T2DM risk. Unfortunately, the 1 -h plasma glucose concentration was not measured in the present study. The cross sectional nature of the study is another limitation. Future recall of the participants in the present cohort for a repeat OGTT will provide longitudinal information about the predictive power of the HbAlc and OGTT in Qatari individuals.

Other limitations of the study include: first, the study has cross sectional design without longitudinal followup. Second, only single OGTT was performed to diagnose glucose tolerance. Third, HOMA-B was utilized to measure beta cell function.

\section{Conclusion}

In this high risk population for T2DM, the OGTT is more sensitive tool than $\mathrm{HbA1c}$ for the identification of subjects with prediabetes. The OGTT and HbA1c identify distinct populations as having high risk of diabetes. However, subjects identified as high risk of diabetes with the OGTT alone have more severe metabolic phenotype

Table 4 Beta cell function (measured with HOMA Model [19] and prevalence of metabolic syndrome according to ATPIII criteria [20] in subjects discordant and concordant for the diagnosis of glucose tolerance with OGT and HbA1c

\begin{tabular}{|c|c|c|c|c|c|}
\hline & Normal by Both Criteria & Prediabetes by Both Criteria & Prediabetes OGTT only & Prediabetes HbA1c Only & $P$-Value \\
\hline Number & 100 & 150 & 146 & 50 & \\
\hline FPG (mg/dl) & $5.2 \pm 0.03$ & $6.0 \pm 0.06$ & $5.9 \pm 0.04$ & $5.3 \pm 0.04$ & $<0.0001$ \\
\hline 2-h PG (mg/dl) & $5.7 \pm 0.1$ & $8.0 \pm 0.2$ & $7.2 \pm 0.2$ & $5.9 \pm 0.1$ & $<0.0001$ \\
\hline $\mathrm{HbA1c}(\%)$ & $6.00 \pm 0.02$ & $5.31 \pm 0.03$ & $5.35 \pm 0.02$ & $5.91 \pm 0.03$ & $<0.0001$ \\
\hline Triglycerides (mM) & $1.41 \pm 0.08$ & $1.72 \pm 0.07$ & $1.68 \pm 0.09$ & $1.62 \pm 0.10$ & $<0.05$ \\
\hline $\mathrm{HDL}(\mathrm{mM})$ & $1.21 \pm 0.03$ & $1.08 \pm 0.02$ & $1.09 \pm 0.02$ & $1.05 \pm 0.03$ & NS \\
\hline HOMA-IR & $3.0+0.3$ & $4.1+0.4$ & $3.1 \pm 0.3$ & $3.6 \pm 0.3$ & $<0.05$ \\
\hline Beta Cell Function & $55 \pm 1$ & $33 \pm 1$ & $34 \pm 1$ & $51 \pm 2$ & $<0.0001$ \\
\hline Stern Score & $8.3 \pm 0.6$ & $22.5 \pm 1.4$ & $16.1 \pm 11$ & $9.8 \pm 0.9$ & $<0.0001$ \\
\hline$\%$ MS & 20 & 58 & 62 & 20 & $<0.0001$ \\
\hline
\end{tabular}

FPG fasting plasma glucose, 2-h PG 2-h plasma glucose, MS metabolic syndrome 
than those identified with HbA1c. Thus, OGTT should be favored over HbAlc in Qatar Diabetes Strategy to screen high risk individuals for intervention programs to reduce their future T2DM risk.

\section{Abbreviations}

ADA: American Diabetes Association; CVD: Cardiovascular disease; FPG: Fasting plasma glucose; HbA1c: Glycated hemoglobin; HOMA: Homeostasis model assessment; IFG: Impaired fasting glucose; IGT: Impaired glucose tolerance; NGT: Normal glucose tolerance; T2DM: Type 2 diabetes mellitus

\section{Acknowledgments}

The Authors would like to thank Mr. Saad Sherida Al Kaabi for his support through the study, Jessie Lirazan RN, and Huda Mejri for their excellent care of the patients during the study.

\section{Authors' contributions}

$\mathrm{SI}, \mathrm{AM}, \mathrm{MJ}$, and $\mathrm{OM}$. have generated the data, DK helped in data analysis, MAG designed the study, contributed to data generation and data analysis, and wrote the first draft of the manuscript. RAD, MZ, and AJ reviewed and revised the manuscript. MAG is the guarantor of this work and, as such, had full access to all the data in the study and takes responsibility for the integrity of the data and the accuracy of the data analysis. All authors read and approved the final manuscript.

\section{Funding}

This study was supported in part by Qatar Foundation grant NPRP 4-248-3076. The funding source had no role in the design, execution of the study or manuscript writing.

\section{Availability of data and materials}

The datasets used and/or analyzed during the current study are available from the corresponding author on reasonable request.

\section{Ethics approval and consent to participate}

The study protocol was approved by the Board of the Ethical Committee of Hamad Medical Corporation (HMC ethical committee) and informed written consent was obtained from participants prior to enrollment.

\section{Consent for publication}

Not applicable.

\section{Competing interests}

The authors declare that they have no competing interests.

\section{Author details}

'Qatar Petroleum, Doha, Qatar. ${ }^{2}$ Academic Health System, Hamad General Hospital, PO Box 3050, Doha, Qatar. ${ }^{3}$ Diabetes Division, University of Texas Health Science Center, San Antonio, TX, USA

Received: 8 August 2018 Accepted: 19 July 2019

Published online: 22 August 2019

\section{References}

1. Shaw JE, Sicree RA, Zimmet PZ. Global estimates of the prevalence of diabetes for 2010 and 2030. Diabetes Res Clin Pract. 2010;87:4-14.

2. Menke A, Casagrande S, Geiss L, Cowie C. Prevalence of and trends in diabetes among adults in the United States, 1988-2012. JAMA. 2015;314: 1021-9.

3. Jaber LA, Brown MB, Hammad A, Nowak SN, Zhu Q, Ghafoor A, Herman WH. Epidemiology of diabetes among Arab Americans. Diabetes Care. 2003; 26:308-13.

4. Bener A, Zirie M, Janahi IM, Al-Hamaq AO, Musallam M, Wareham NJ. Prevalence of diagnosed and undiagnosed diabetes mellitus and its risk factors in a population-based study of Qatar. Diabetes Res Clin Pract. 2009:84:99-106.

5. al-Mahroos F, McKeigue PM. High prevalence of diabetes in Bahrainis. Associations with ethnicity and raised plasma cholesterol. Diabetes Care. 1998;21:936-42
6. DeFronzo RA, Tripathy D, Schwenke DC, Banerji M, Bray GA, Buchanan TA, Clement SC, Henry RR, Hodis HN, Kitabchi AE, Mack WJ, Mudaliar S, Ratner RE, Williams K, Stentz FB, Musi N, Reaven PD, ACT NOW Study. Pioglitazone for diabetes prevention in impaired glucose tolerance. N Engl J Med. 2011; 364:1104-15.

7. DREAM (Diabetes REduction Assessment with ramipril and rosiglitazone Medication) Trial Investigators, Gerstein HC, Yusuf S, Bosch J, Pogue J, Sheridan P, Dinccag N, Hanefeld M, Hoogwerf B, Laakso M, Mohan V, Shaw J, Zinman B, Holman RR. Effect of rosiglitazone on the frequency of diabetes in patients with impaired glucose tolerance or impaired fasting glucose: a randomised controlled trial. Lancet. 2006:368:1096-105.

8. Knowler WC, Barrett-Connor E, Fowler SE, Hamman RF, Lachin JM, Walker EA, Nathan DM, Diabetes Prevention Program Research Group. Reduction in the incidence of type 2 diabetes with lifestyle intervention or metformin. $\mathrm{N}$ Engl J Med. 2002;346:393-403.

9. Abdul-Ghani MA, DeFronzo RA. Plasma glucose concentration and prediction of future risk of type 2 diabetes. Diabetes Care. 2009; 32(Suppl 2):S194-8.

10. Abdul-Ghani MA, Tripathy D, DeFronzo RA. Contributions of beta-cell dysfunction and insulin resistance to the pathogenesis of impaired glucose tolerance and impaired fasting glucose. Diabetes Care. 2006;29: 1130-9.

11. American Diabetes Association. Prevention or delay of type 2 diabetes. Diabetes Care 2018:41:S44-54.

12. International Expert Committee. International expert committee report on the role of the A1C assay in the diagnosis of diabetes. Diabetes Care. 2009; 32:1327-34

13. Ehehalt S, Wiegand S, Körner A, Schweizer R, Liesenkötter KP, Partsch CJ, Blumenstock G, Spielau U, Denzer C, Ranke MB, Neu A, Binder G, Wabitsch M, Kiess W, Reinehr T. Low association between fasting and OGTT stimulated glucose levels with $\mathrm{HbA1C}$ in overweight children and adolescents. Pediatr Diabetes. 2017:18(8):734-41 [Epub ahead of print].

14. Jackson SL, Safo SE, Staimez LR, Olson DE, Narayan KMV, Long Q, Lipscomb J. Rhee MK, Wilson PWF, Tomolo AM, Phillips LS. Glucose challenge test screening for prediabetes and early diabetes. Diabet Med. 2017;34:716-24.

15. Eastwood SV, Tillin T, Sattar N, Forouhi NG, Hughes AD, Chaturvedi N. Associations between prediabetes, by three different diagnostic criteria, and incident CVD differ in south Asians and Europeans. Diabetes Care. 2015;38: 2325-3.

16. van Valkengoed IG, Vlaar EM, Nierkens V, Middelkoop BJ, Stronks K. The uptake of screening for type 2 diabetes and prediabetes by means of glycated hemoglobin versus the Oral glucose tolerance test among 18 to 60-year-old people of south Asian origin: a comparative Study. PLoS One. 2015;10:e0136734

17. Eastwood SV, Tillin T, Mayet J, Shibata DK, Wright A, Heasman J, Beauchamp N, Forouhi NG, Hughes AD, Chaturvedi N. Ethnic differences in crosssectional associations between impaired glucose regulation, identified by oral glucose tolerance test or HbA1c values, and cardiovascular disease in a cohort of European and south Asian origin. Diabet Med. 2016:33:340-7.

18. Li J, Ma H, Na L, Jiang S, Lv L, Li G, Zhang W, Na G, Li Y, Sun C. Increased hemoglobin A1c threshold for prediabetes remarkably improving the agreement between $\mathrm{A} 1 \mathrm{C}$ and oral glucose tolerance test criteria in obese population. J Clin Endocrinol Metab. 2015;100:1997-2005.

19. Matthews DR, Hosker JP, Rudenski AS, Naylor BA, Treacher DF, Turner RC. Homeostasis model assessment: insulin resistance and beta-cell function from fasting plasma glucose and insulin concentrations in man. Diabetologia. 1985;28:412-9.

20. National Cholesterol Education Program (NCEP) expert panel on detection, evaluation, and treatment of high blood cholesterol in adults (adult treatment panel III). Third report of the National Cholesterol Education Program (NCEP) expert panel on detection, evaluation, and treatment of high blood cholesterol in adults (adult treatment panel III) final report. Circulation. 2002;106:3143-21.

21. Stern MP, Williams K, Haffner SM. Identification of persons at high risk for type 2 diabetes mellitus: do we need the oral glucose tolerance test? Ann Intern Med. 2002;136:575-81.

22. The Expert Committee on the Diagnosis and Classification of Diabetes Mellitus. Report of the expert committee on the diagnosis and classification of diabetes mellitus. Diabetes Care. 1997;20:1183-97.

23. DeFronzo RA, Abdul-Ghani MA. Preservation of beta-cell function: the key to diabetes prevention. J Clin Endocrinol Metab. 2011;96:2354-66. 
24. Pinelli NR, Jantz AS, Martin ET, Jaber LA. Sensitivity and specificity of glycated hemoglobin as a diagnostic test for diabetes and prediabetes in Arabs. J Clin Endocrinol Metab. 2011;96:E1680-3.

25. Kanat M, Winnier D, Norton $L$, Arar N, Jenkinson C, DeFronzo RA, AbdulGhani MA. The relationship between beta cell function and glycated hemoglobin. Diabetes Care. 2011;34:1006-10.

26. Grundy SM. Pre-diabetes, metabolic syndrome, and cardiovascular risk. J Am Coll Cardiol. 2012;59:635-43.

27. Abdul-Ghani MA, Lyssenko V, Tuomi T, DeFronzo RA, Groop L. Fasting versus post load plasma glucose concentration and the risk for future type 2 diabetes: results from the Botnia study. Diabetes Care. 2009;32:281-6.

28. Abdul-Ghani MA, Abdul-Ghani TA, Ali N, DeFronzo RA. One hour plasma glucose concentration and the metabolic syndrome identify subjects at high risk for future type 2 diabetes. Diabetes Care. 2008;31:1650-5.

\section{Publisher's Note}

Springer Nature remains neutral with regard to jurisdictional claims in published maps and institutional affiliations.

Ready to submit your research? Choose BMC and benefit from:

- fast, convenient online submission

- thorough peer review by experienced researchers in your field

- rapid publication on acceptance

- support for research data, including large and complex data types

- gold Open Access which fosters wider collaboration and increased citations

- maximum visibility for your research: over $100 \mathrm{M}$ website views per year

At $\mathrm{BMC}$, research is always in progress.

Learn more biomedcentral.com/submissions 